\title{
PREVALENCE OF HEPATITIS B AND CARRIER RATE IN GENERAL POPULATION AT A TERTIARY CARE HOSPITAL
}

\author{
Supriya Christopher ${ }^{1}$, P. M. Giridhara Upadhyaya ${ }^{2}$
}

${ }_{1}^{1}$ Associate Professor, Department of Microbiology, KIMS, Bangalore.

${ }^{2}$ Associate Professor, Department of Microbiology, KIMS, Bangalore.

ABSTRACT
BACKGROUND
Hepatitis B is a disease that has been gaining more and more interest of late with a lot of research being conducted on its
pathogenicity, its structure, the epidemiology of the disease and prospects and outcomes of vaccination. It has captured so much
attention because of its grievous consequences and widespread prevalence as well as the ease with which it can be controlled.
Our objective was to assess trends in the prevalence of hepatitis B virus (HBV) infection in a tertiary care hospital in Bangalore
and to determine the HBV carrier rate, magnitude and pattern of HBV infected super carriers and simple carriers in patients
positive for hepatitis B surface antigen.

\section{MATERIALS AND METHODS}

This is a hospital-based prospective observational study. The prevalence of HBV infection was determined in a representative sample of the general population. It was a hospital-based prospective study. Study period being 2 months, June - August 2015 with sample size of 2061 samples.

Inclusion Criteria: Samples taken randomly during inpatient routine screening, outpatient screening or blood bank donor screening.

Exclusion Criteria: Subjects suffering from present liver disease or HIV positive at point of blood collection.

\section{RESULTS}

The total number of samples tested for HBsAg by the chemiluminescence method was 2061, out of which 29 samples were found to be HBsAg carriers satisfying the inclusion and exclusion criteria. This gives the gross rate of positivity as $[29 / 2061] * 100=1.41 \%$. The studies on prevalence HBV 2 out of the $29 \mathrm{HBsAg}$ positive individuals tested positive for HBeAg, giving a percentage of $6.89 \%$. This indicates a state of active HBV DNA synthesis and high infectivity (Super carrier).

\section{CONCLUSION}

HBsAg have given results which are consistent in their inconsistency, but that is probably due to the vast and diverse nature of populations across India. However, we can conclude that the rate is still very high compared to the rest of the world, especially first world countries. Thus, any preventive measures which are applied throughout the country must also be tailored to fit the needs of specific regions if they are to be truly effective.

\section{KEYWORDS}

HBV, Carrier, Prevalence, Vaccine.

HOW TO CITE THIS ARTICLE: Christopher S, Upadhyaya PMG. Prevalence of hepatitis B and carrier rate in general population at a tertiary care hospital. J. Evolution Med. Dent. Sci. 2018;7(03):356-360, DOI: 10.14260/jemds/2018/79

\section{BACKGROUND}

Hepatitis B is a disease that has been gaining more and more interest of late with a lot of research being conducted on its pathogenicity, its structure, the epidemiology of the disease and prospects and outcomes of vaccination. It has captured so much attention because of its grievous consequences and widespread prevalence as well as the ease with which it can be controlled. Trends in research in Hepatitis $B$ have only picked up pace recently due to the increasing prevalence and increasing awareness of the fatal sequel of this disease. Although, the modes of transmission and threat level of the disease are similar to HIV, Hepatitis B has not had as

'Financial or Other Competing Interest': None.

Submission 07-12-2017, Peer Review 01-01-2018,

Acceptance 06-01-2018, Published 13-01-2018.

Corresponding Author:

Dr. Supriya Christopher,

Associate Professor,

Department of Microbiology,

KIMS, Banashankri $2^{\text {nd }}$ Stage, Bangalore-560070.

E-mail: supriyachristopher@hotmail.com

DOI: $10.14260 /$ jemds $/ 2018 / 79$ explosive an introduction into the minds of the public, probably due to its unpredictable progression with some being unaware that they have been infected, while others die of hepatocellular carcinoma. Around the world, it has been estimated that nearly 350 - 400 million people have been infected by HBV. HBV related chronic infections result in nearly $60 \%$ cases of cirrhosis and $80 \%$ cases of hepatocellular carcinoma with end-stage liver disease accounting for one for every 40 deaths. ${ }^{1}$ The incubation period for hepatitis B is 45 - 160 days, in which period transfer of the virus may occur to countless individuals, thus perpetuating the cycle of infection. Moreover, HBV is about a hundred times more infectious than the human immunodeficiency virus (HIV) and is easier to transmit by needle-stick injuries. ${ }^{2}$

Most scientists agree that apart from the presence of HBsAg, the presence of HBeAg indicates high infectivity, known as 'Super carriers' or 'Active carriers,' and the absence of $\mathrm{HBeAg}$ along with presence of anti-Hepatitis B e-antigen (Anti-HBe) indicates the low infectivity 'Simple carrier' state or 'inactive/ healthy carriers.' 3,4 
The total HBV carrier pool in India is estimated to be around 43 million. Out of the estimated 400 million infected around the world, this gives India the dubious distinction of having one-tenth of all carriers worldwide. ${ }^{5}$ Around 1 million new HBV carriers are added to this pool annually. ${ }^{6}$ The average estimated carrier rate of $\mathrm{HBV}$ in India is $4.7 \%{ }^{7}$ However, there have been vast inconsistencies and differences among different studies in India with different studies exhibiting a varying seroprevalence of $0.8 \%$ to $42.5 \%{ }^{8,9}$

The WHO estimates that around 55 percent of children around the world receive 3 doses of vaccine. ${ }^{10}$ Coverage around the world and in India has remained low and targets only very small subpopulations.11,12 We are progressing towards greater coverage for children by the inclusion of the Hepatitis B vaccine in the Universal Immunisation Program instituted by the Indian Academy of Paediatrics, but its impact remains yet to be seen in subsequent cohorts of vaccinated children. ${ }^{13}$

The estimation of the number of carriers in most studies is mostly done by the detection of the HBsAg in individuals, but the detection of the presence of the HBeAg and anti-HBe has not yet gained mainstream attention. This study aims to estimate the carrier rate and pattern at a tertiary care hospital in Bangalore by detecting the presence HBsAg by the chemiluminescence method, and the presence of HBeAg and the anti-HBe (IgG) among the carriers by the rapid immunochromatographic assay method.

\section{MATERIALS AND METHODS}

This is a hospital-based prospective observational study. All the blood samples were taken from samples that came in for routine screening at the Department of Microbiology and Hospital Blood Bank at the Tertiary Care Hospital. The samples were collected over a period of 45 days in the months of June - July 2015.

A $5 \mathrm{~mL}$ tube of whole blood was collected following standard procedures using a sterile container with no anticoagulant added from each patient. Samples were allowed to clot for one hour at room temperature. It was then centrifuged for 10 minutes at approximately $1000 \mathrm{~g}$. An aliquot of $500 \mu \mathrm{L}$ of serum was made into labelled cryo vials using clean pipette technique. Samples being tested immediately were stored at 4 degrees. Long term storage was done by freezing vials of serum in a deep freezer. Any time a serum sample was taken out of deep freeze. It was recentrifuged before storage.

Samples were tested for HBsAg using automated chemiluminescence method [HBsAg Assay II, Cobas® ${ }^{\circledR}$ E601, Roche Diagnostics, Germany]. The test works on the sandwich principle and takes a total duration of 18 minutes. In the 1st incubation period, 50 microlitres of sample, two monoclonal Anti-HBsAg antibodies and a mix of monoclonal and polyclonal antibodies labelled with a ruthenium complex form a sandwich complex. During the second incubation, the complex gets bound to the solid phase after the addition and interaction of streptavidin-coated microparticles. This reaction mix is aspirated into a measuring cell where the microparticles are captured onto an electrode surface through magnetism. The unbound substances get removed and an electric current is passed through the electrode. This causes a chemiluminescent reaction, which is detected and measured by a photomultiplier.

Samples testing positive for HBsAg and meeting the inclusion and exclusion criteria were considered as HBsAg positives and were stored in deep freeze in labelled cryo vials.

At the end of the experimental collection period, the samples positive for HBsAg which were stored were then tested for HBeAg and Anti-HBe antibody IgG using rapid immunochromatographic assay kit [Insight Device, Tulip Diagnostics, India]. The Anti-HBe IgG was tested as IgG is an indicator of long-standing old HBV infection, whereas the IgM signified present infection. (Rapid immunochromatographic assay was used instead of enzyme-linked immunosorbent assay due to laboratory availability and cost factors).

The Insight HBeAg test is a rapid, self-performing, immunochromatographic assay for the detection of inhuman serum. Sensitivity of HBeAg is $1 \mathrm{ncu} / \mathrm{mL}$ and Insight Anti-Hbe IgG test is a rapid, competitive, self-performing, immunochromatographic assay for the detection of $\mathrm{HBeAg}$ IgG in human serum. Sensitivity of Anti-Hbe IgG is $4 \mathrm{ncu} / \mathrm{mL}$.

The two tests are based on the principle of serum agglutination on a membrane. For the HBeAg test, the conjugate pad contains Anti-HBe-colloid gold and mouse IgGcolloid gold. As the test specimen flows through the device, the Anti-HBe-colloid gold conjugate complexes with the HBeAg as well as the mouse IgG-colloid gold and travels further along the cassette to get immobilised by Anti-HBe and form a pink band at the test region. The mouse IgG-colloid gold and unbound Anti-HBe move further to the Control region, where it forms a band and serves as control. The test for the Anti-HBe is similar but uses specific antibodies to the IgG instead. Samples positive for HBsAg and HBeAg were designated Super carriers. Those positive for HBsAg and AntiHBe IgG, with absence of HBeAg were designated Simple carriers. All patients were advised to get their families vaccinated against Hepatitis B.

Data was collated using the immunological tests and hospital data. All obtained data was used to determine rate of positivity for HBsAg and pattern (susceptible groups) of occurrence of HBsAg with regards to age and sex as well as the presence of HBeAg and Anti-HBe IgG to determine the percentage of Super carriers and Simple carriers. Data was entered into Excel and analysed with descriptive and inferential statistics.

Quality control was as per standard procedure recommended by MCI. All testing was conducted with informed consent and strict confidentiality has been maintained. Ethical clearance was obtained from the Institutional Ethics Committee after review of the study proposal.

\section{RESULTS}

After immunological testing and statistical analysis of the data, the following observations were seen.

\section{Gross Carrier Rate}

The total number of samples tested for HBsAg by the chemiluminescence method was 2061, out of which 29 samples were found to be HBsAg carriers satisfying the inclusion and exclusion criteria. This gives the gross rate of positivity as: $[29 / 2061]^{*} 100=1.41 \%$. 


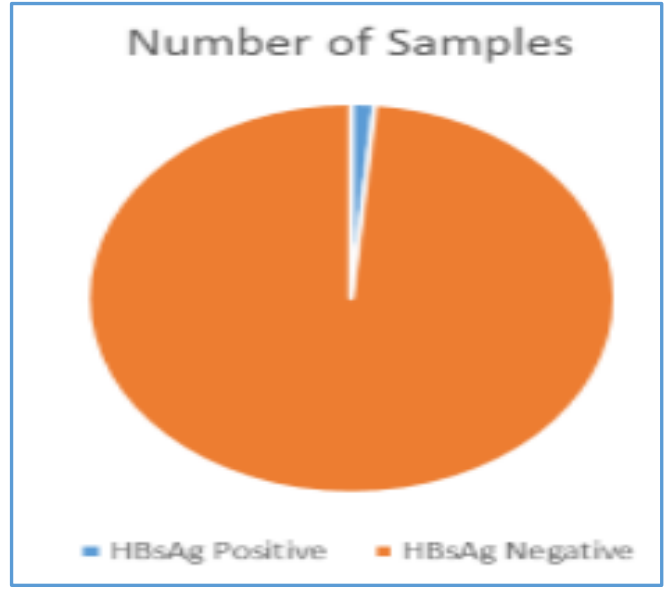

Figure 1

\section{Gender-Wise distribution of HBsAg Carriers}

The gender-wise distribution of the positivity of HBsAg is as follows. There was a majority of male patients being tested with a female-to-male ratio of 485: 1596

\begin{tabular}{|c|c|c|c|c|}
\hline Gender & $\begin{array}{c}\text { Total } \\
\text { Number of } \\
\text { Samples }\end{array}$ & $\begin{array}{c}\text { Percentage } \\
\text { of Total } \\
\text { Samples }\end{array}$ & Positive & $\begin{array}{c}\text { HBsAg } \\
\text { Positive } \\
\text { Percentage }\end{array}$ \\
\hline Male & 1596 & $77.44 \%$ & 24 & $1.50 \%$ \\
\hline Female & 485 & $23.53 \%$ & 5 & $1.03 \%$ \\
\hline \multicolumn{5}{|r|}{ Table 1. The Findings of the Gender-Wise Distribution are } \\
Represented
\end{tabular}

\section{Age-Wise distribution of HBsAg Carriers}

The $29 \mathrm{HBsAg}$ positive samples were sorted according to age groups by decade and the following observations were seen There were no positives in the under 20 years' group. The age range of the positive samples was 24 years to 80 years of age.

\begin{tabular}{|c|c|c|}
\hline $\begin{array}{c}\text { Age Group } \\
\text { (Years) }\end{array}$ & $\begin{array}{c}\text { Number of } \\
\text { Carriers }\end{array}$ & $\begin{array}{c}\text { Percentage of } \\
\text { Total Carriers }\end{array}$ \\
\hline $0-10$ & 0 & $0.0 \%$ \\
\hline $11-20$ & 0 & $0.0 \%$ \\
\hline $21-30$ & 7 & $24.14 \%$ \\
\hline $31-40$ & 10 & $34.48 \%$ \\
\hline $41-50$ & 1 & $3.45 \%$ \\
\hline $51-60$ & 2 & $6.89 \%$ \\
\hline $61-70$ & 7 & $24.14 \%$ \\
\hline $71-80$ & 2 & $6.89 \%$ \\
\hline \multicolumn{3}{|c|}{ Table 2 } \\
\hline
\end{tabular}

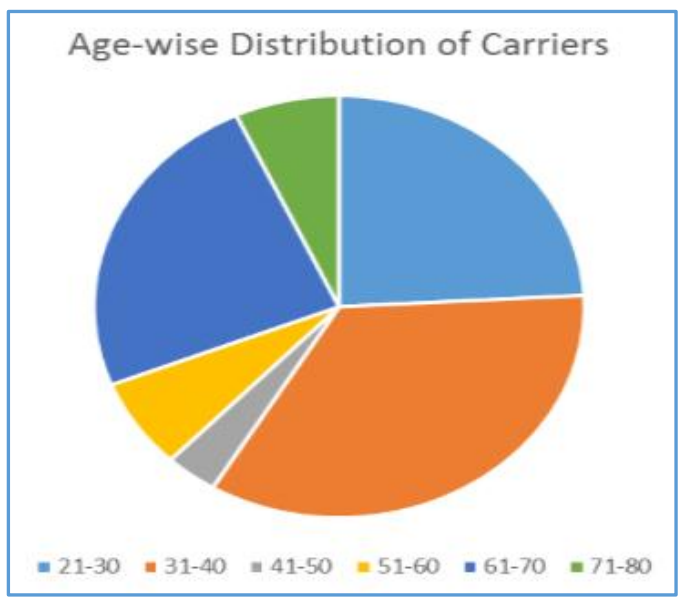

Figure 2

\section{Super Carriers and Simple Carriers}

Following the identification of the 29 carriers, they were tested for HBeAg and Anti-HBe IgG. They were designated as simple carrier if they were HBsAg positive, HBeAg negative and Anti-HBe positive. They were designated as super carrier if they were HBsAg positive, HBeAg positive and Anti-HBe negative.

The following table shows the number of HBsAg positive samples sorted according to the type of carrier.

\begin{tabular}{|c|c|c|}
\hline Type of Carrier & Number of Carriers & Percentage \\
\hline Simple carrier & 27 & $93.10 \%$ \\
\hline Super carrier & 2 & $6.89 \%$ \\
\hline \multicolumn{3}{|c|}{ Table 3 } \\
\hline
\end{tabular}

Out of the two carriers testing positive for $\mathrm{HBeAg}$, one was male (age 52 years) and the other was female (age 63 years).

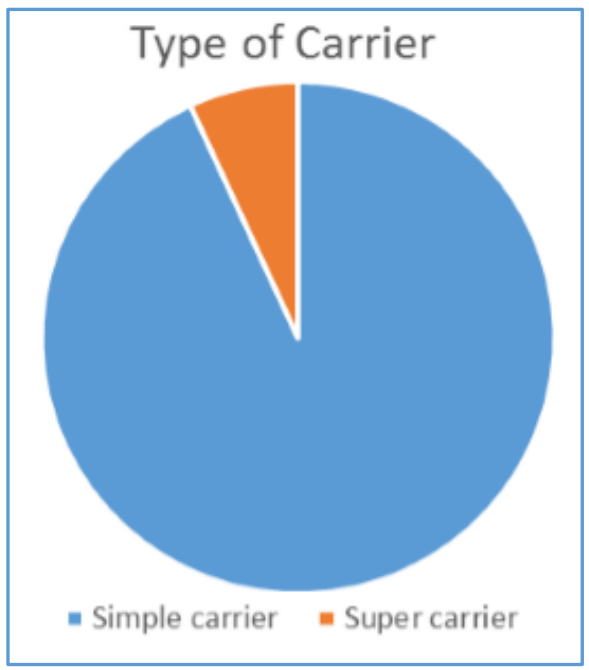

Figure 3

\section{DISCUSSION}

Hepatitis B is a communicable disease with a heavy burden on any society and carriers pose a great risk to the population. It is especially significant for public health as it is easily vaccine preventable, but sufficient measures are not being taken to combat this potentially deadly disease. As one of the leading causes of hepatocellular disease and liver carcinoma, ${ }^{1}$ it cannot be ignored and requires comprehensive strategies for its identification and control.

In our study we have found that out of a pool of 2061 individuals, 29 were found to be carriers of HBsAg, which gives a gross carrier percentage of $1.41 \%$. This result is a lower figure than the quoted national average carrier rate of $4.7 \%$.6] However, this lower than average value may be due to the fact that this study was conducted in an urban population in a major city, which does not have endemicity. A study by Batham et al showed a marked variation between tribal and non-tribal (urban) infectivity percentages $(15.9 \%$ and $2.4 \%$ respectively), so the lower than average result tallies with the observation. ${ }^{8}$ Other studies in population in major cities have shown carrier percentages of as low as $0.61 \%$ and $0.87 \%$ in India. 7,14

As can be seen from Table 1 there was an approximate 1:3 ratio of females-to-males in the total samples tested, but the percentage of HBsAg positive in females and males was 
$1.03 \%$ and $1.50 \%$ respectively. This result showed that although the number of samples tested was different, the carrier percentage of males and females was relatively the same. The larger number of male samples was probably due to the fact that most voluntary donors at the blood bank was male.

The age-wise segregation of HBsAg positive individuals showed that a maximum percentage of individuals belonged to the 31 - 40 years' age group (34.48\%) as seen in Figure 2 . This was followed by 21 - 30 years' and $61-70$ years' age group with $24.14 \%$ each. Following this was the $51-60$ years' and 71 - 80 years' group with $6.89 \%$, and lastly the 41 50 groups formed $3.45 \%$ of the total carrier pool. The higher prevalence in the $31-40$ age group may be due to unprotected sexual contact and unsafe needle sharing practices. However, the familial contact may also be a big contributor. ${ }^{3}$ The relatively older age groups being tested positive may be due to the fact that a couple of decades are required for the development of chronicity of hepatitis $B$ infection.

An interesting find was that no HBsAg positive individuals below 20 years were found in the study period. This may be because of the HBV vaccines that have been rolled out since 2007 in India. Karnataka was one of the first ten states in which the vaccine policy was implemented, so it stands to reason that the policy is probably being implemented effectively. ${ }^{11}$ Contrastingly, other studies have shown a high rate of HBsAg positivity in children under 10 years as well which indicates that this may be a region specific result. ${ }^{11}$ Moreover studies in India show a high rate of HBV infection in pregnant women, so perinatal transmission is still a major route of infection, but regular antenatal screening and birth dose of HBV vaccine seem to be effective in preventing its vertical transmission. ${ }^{13}$

Following HBsAg, 2 out of the 29 HBsAg positive individuals tested positive for $\mathrm{HBeAg}$ as seen in Table 3 giving a percentage of $6.89 \%$. The individuals were one male and one female. This indicates a state of active HBV DNA synthesis and high infectivity (Super carrier). ${ }^{2}$ Some other studies have shown $\mathrm{HBeAg}$ positivity rates ranging from $1 \%$ to $17.27 \%$ in various subpopulations. Most studies still use HBeAg assay only for prognostic value or for determining HBV DNA synthesis activity, but it would be helpful to also classify carriers based on their infectivity when trying to determine cost effective measures of prevention and treatment of Hepatitis B. 15,16,17,18

The assessment of HBeAg and infectivity status has practical uses in transfusion centres for counselling HBsAg positive blood donors, for determining priority targets of immunisation and advising the medical professionals on the care and precautions during the care of high infectivity carriers.

However, as with any study this study is also limited by the fact that it only assesses one major tertiary care hospital in Bangalore, Karnataka. This limits the population studied to an urban one, which affects prevalence rates no doubt. However, this result is useful for further epidemiological studies and to study the effectiveness of any further measures to combat Hepatitis B.

Thus, we can see that although many measures are in place to combat the spread of Hepatitis B, there are still many carriers hidden in the population. This indicates that preventive measures have to be stepped up and implemented in more widespread.

\section{CONCLUSION}

Hepatitis B is a major public health issue in India and around the world. Ever since its emergence into importance 30 - 40 years ago, many efforts have been made to combat it and curb its spread. With devastating consequences like hepatocellular carcinoma and liver cell death, especially seen among the children who get infected, it is a great burden on our society. The main point of contention is its easy spread with a very minute viral load, by means as simple as saliva, mucus or even sweat.

Although, the main modes of spread continue to be parenteral routes like through mother to child, tainted blood from blood banks, unprotected sexual contact and injectable drug use, measures need to be put in place to ensure that high infectivity individuals are isolated and made non-infectious as soon as possible.

The studies on prevalence of HBsAg have given results which are consistent in their inconsistency, but that is probably due to the vast and diverse nature of populations across India. However, we can conclude that the rate is still very high compared to the rest of the world, especially first world countries. Thus, any preventive measures which are applied throughout the country must also be tailored to fit the needs of specific regions, if they are to be truly effective.

As Hepatitis B is very easy to prevent but difficult to treat, the emphasis of any national policy should be on vaccination and prevention rather than treatment. Efforts have been made by our Government with the introduction of compulsory Hepatitis B vaccination under the Universal Immunization Program, but the implementation of any program in India remains sporadic and disproportionately skewed.

More emphasis also needs to be made on adult vaccination, especially among healthcare workers, high-risk groups and those in endemic areas as well as education on safe practices for food handlers, blood banks and promiscuous individuals. The only way we can lessen the burden of this disease is by constant vigilance for its prevention.

This study showed $1.41 \%$ of individuals are carriers of HBsAg and among them $6.89 \%$ are positive for HBeAg. The 31 to 40 age group formed a majority of the positive samples with $34.48 \%$ of the positive samples \%. There was no significant difference among male and female prevalence rates. This result shows that the prevalence of Hepatitis individuals are carriers of HBsAg and among them $6.89 \%$ are positive for HBeAg. The 31 to 40 age group formed a majority of Hepatitis B is still very high and carriers with high infectivity must be treated with extra caution and care to help prevent the spread of this disease.

\section{REFERENCES}

[1] Koff RS. Review article: vaccination and viral hepatitiscurrent status and future prospects. Aliment Pharmacol Ther 2007;26(10):1285-92.

[2] Viral Hepatitis Prevention Board. Antwerp VHPB Report. Editorial. Control of viral hepatitis in Europe. Viral Hepatitis 1996;4(2). 
[3] Gerlich WH. Medical virology of hepatitis B: how it began and where we are now. Virol J 2013;10:239.

[4] Dow BC, Macvarish I, Barr A, et al. Significance of tests for HBeAg and anti-HBe in HBsAg positive blood donors. J Clin Pathol 1980;33(11):1106-9.

[5] World Health Organisation. Children's Vaccines-Safety First. Geneva: WHO; 1999.

[6] Batham A, Narula D, Toteja T, et al. Sytematic review and meta-analysis of prevalence of hepatitis B in India. Indian Pediatr 2007;44(9):663-74.

[7] Arankalle VA, Chadha MS, Gandhe SS. HBsAg positivity among rural populations of Pune (1982-1998). Indian J Gastroenterol 1999;18(Suppl 1):S27.

[8] Rajani M, Jais M. Magnitude and pattern of hepatitis B infection in clinically suspected infectious hepatitis at a tertiary care hospital in Urban India. J Glob Infect Dis 2014;6(3):105-8.

[9] "Hepatitis B." WHO.Web.7Oct.2015.<http://www.who.int/mediacen tre/factsheets/fs204/en/>.

[10] Margolis HS, Alter MJ, Hadler SC. Hepatitis B: evolving epidemiology and implications for control. Semin Liver Dis 1991;11(2):84-92.

[11] Lahariya C, Subramanya BP, Sosler S. An assessment of hepatitis B vaccine introduction in India: lessons for roll out and scale up of new vaccines in immunization programs. Indian J Public Health 2013;57(1):8-14.

[12] "Indian Pediatrics-Editorial." Indian Pediatrics$\begin{array}{llll}\text { Editorial. Web. } & 7 & \text { Oct. } & 2015 .\end{array}$ <http://indianpediatrics.net/nov2001/nov-13351338.htm>.
[13] Banerjee A, Chakravarty R, Mondal PN, et al. Hepatitis $B$ virus genotype $D$ infection among antenatal patients attending a maternity hospital in Calcutta, India: assessment of infectivity status. Southeast Asian J Trop Med Public Health 2005;36(1):203-6.

[14] Prasad M, Thomas R, Devaraj. Prevalence of hepatitis $B$ virus in acute hepatitis in south India. Indian J of Gastroenterol 1999.

[15] Sacher M, Unger FD, Eder G. Vertical hepatitis B transmission by anti-HBe positive hepatitis B carrier mothers. Padiatr Padol 1986;21(2):141-6.

[16] Okada K, Kamiyama I, Inomata M, et al. E antigen and anti-e in the serum of asymptomatic carrier mothers as indicators of positive and negative transmission of hepatitis $\mathrm{B}$ virus to their infants. $\mathrm{N}$ Engl J Med 1976;294(14):746-9.

[17] Lamtha SC, Dixit VK, Jain AK, et al. Correlation of age and hepatitis B virus DNA levels with histological activity in HBeAg negative chronic hepatitis B patients. Trop Gastroenterol 2014;35(3):152-6.

[18] Seiz PL, Slanina H, Ziebuhr J, et al. Studies of nosocomial outbreaks of hepatitis B in nursing homes in Germany suggest a major role of hepatitis B e antigen expression in disease severity and progression. Int J Med Microbiol 2015;305(7):663-72. 